\title{
Pressure Control Method of an Electro-hydraulic Actuated Clutch Considering Hysteresis
}

\author{
Rong Zeng ${ }^{1,2}$, Long Xue ${ }^{3}$ and Jun Xiao ${ }^{4, *}$ \\ ${ }^{I}$ College of Engineering, Huazhong Agricultural University, Wuhan 430070, China \\ ${ }^{2}$ Key Laboratory of Agricultural Equipment in Mid-lower Yangtze River, Ministry of Agriculture, Wuhan 430070, China \\ ${ }^{3}$ Dongfeng Motor Group Co., LTD Technology Centre, Wuhan 430058, China \\ ${ }^{4}$ School of Mechanical and Electronic Engineering, Wuhan University of Technology, Wuhan 430070, China
}

Received 11 June 2019; Accepted 14 October 2019

\begin{abstract}
The nonlinear hysteretic characteristics of a wet clutch's pressure control valve in dual clutch transmission (DCT) can cause pressure response delay, which affects pressure control accuracy. This study proposed a compound control method for hysteresis compensation based on the analysis of the nonlinear hysteretic characteristics of the electrohydraulic pressure control valve to reveal the influence mechanism of hysteresis on the wet clutch and to improve pressure control accuracy. The compound control method included a feed-forward controller and an additional feedback controller. The pressure hysteresis of the electrohydraulic pressure control valve was analyzed through mathematical modeling of the valve spool. Two experiments were designed to verify and characterize the pressure hysteresis. The feed-forward control algorithm for hysteresis compensation combined with the proportional-integral-derivative (PID) controller was formulated to realize pressure control based on the characteristics of the wet clutch's pressure hysteresis. The feasibility and effectiveness of the proposed control method were verified through bench tests. Results show that the average absolute error in steady state reduces from 0.186 bar to 0.005 bar under the proposed control method. The average relative error decreases from $2.924 \%$ to $0.092 \%$ compared with the system without any pressure control. In addition, the use of the proposed control method in dynamic working condition reduces the average absolute error from 0.189 bar to 0.043 bar and decreases the average relative error from $2.963 \%$ to $0.722 \%$ compared with the system without any pressure control. The proposed control method under the state with step pressure input can guarantee the system response speed and control accuracy. Finally, the comparison of compound control, feed-forward control, and PID control shows that the compound control method can achieve optimum performance in pressure tracking regardless of the test conditions. This study provides an easy-to-implement and high-precision method for pressure control of automotive wet clutches.
\end{abstract}

Keywords: Wet Clutch, Electrohydraulic Pressure Control Valve, Hysteresis compensation, Compound Control

\section{Introduction}

Wet clutches are widely used in dual clutch transmissions (DCTs) because of their large transmission torque, tight structure, long service life, and easy automatic control. Given the high transmission efficiency and extremely fast shifting speed of DCTs, they greatly enhance the driving performance and economy of vehicles [1], thereby becoming an important tendency for the development of transmission technology. Electrohydraulic actuators are widely used as actuators for wet clutch engagement. The electrohydraulic actuators adjust the pressure of the hydraulic system based on proportional solenoid valves to realize the engagement of wet clutches, thereby transmitting the engine torque to the power trains.

The hydraulic oil medium causes remarkable nonlinearity in the electrohydraulic valve. The compressibility of the hydraulic oil and the frictional damping that acts on the valve spool lead to the time delay of the clutch pressure response, hysteresis, and filling phase

\footnotetext{
*E-mail address: xia0junxijun@163.com
}

ISSN: 1791-2377 @ 2019 School of Science, IHU. All rights reserved.

doi:10.25103/iestr.125.21 dynamics, as well as other nonlinear elements [2]. These nonlinear characteristics introduce a significant challenge to the precise pressure control of wet clutches. The transmitted torque is largely dependent on the pressure of the hydraulic system when clutches are in the slip phase. Therefore, the precise pressure control of wet clutches has become an important issue in improving the quality of DCT shifting.

Present studies on wet clutches mainly focus on the nonlinear characteristics and model building of hydraulic system [3-6]. Most of these studies conduct mathematical analysis on spool dynamics by considering the nonlinearities in hydraulic system, thereby resulting in complexities and remarkable nonlinearities in the established models. Thus, these models are difficult to be achieved in the practical application of production vehicles. As a non-negligible influence factor that influences the movement of the spool, frictional damping leads to hysteresis, which generates the hysteresis phenomenon between the output pressure of the wet clutch and the control current of the electrohydraulic valve, thereby making the formation of a one-to-one correspondence between the actual output pressure and the target pressure impossible to be realized and affecting the pressure control accuracy. Existing pressure control methods for wet clutches are mostly implemented via PID [7], in 
which the deviation of the actual and target pressure is used as the system input. PID control methods cannot realize pressure hysteresis compensation, thereby causing difficulty in attaining high pressure control accuracy. Therefore, establishing a pressure hysteresis compensation method for wet clutches with easy practical engineering application is an urgent problem to be solved to realize precise pressure control.

Consequently, this study analyzes the pressure hysteresis characteristics of a wet clutch's electrohydraulic pressure control valve via theoretical modeling and experimental design. A pressure control algorithm for hysteresis compensation is established to control the wet clutch's output pressure with high accuracy, thereby providing technical support for improving the shifting quality of DCTs.

\section{State of the art}

Many experts and scholars conducted abundant studies on wet clutches and hysteresis control. Losero R. et al. [8] designed a virtual strain gauge where the transmitted torque of the DCT clutch is estimated on the basis of a fuzzy discrete angular domain observer. Certain estimation deviation existed given that the method was based on a linear torsional dynamic model and the dynamic model parameters used in clutch torque estimation were linear. Jiwon J. O. et al. [9] constructed a transmission torque observer for DCT clutch through power train modeling, and the clutch torque was estimated using the vehicle test data. However, the clutch torque in the slip phase was modeled via Coulomb friction model. Thus, the accuracy of the estimated results should be further verified because the friction stage of the clutch varied with many factors. Tarasow A. et al. [10] proposed two kinds of prediction models by employing an adaptive and extended Kalman filter with recursive prediction, and the characteristic parameters of the wet clutch were estimated. The method was based on the state space model of clutch dynamics. Hence, this method depended on the accuracy of the established model. Berkel K. V. et al. [11] designed a clutch engagement controller, which enabled rapid clutch engagement to reduce shifting time, and accompanied with smooth clutch engagement to track the required torque without significant torque drop. The first-order linear dynamic model was applied in the clutch dynamic modeling. Chen M. et al. [12] investigated the torque transmission characteristics of the clutch frictional discs according to the attributes of a multidisc wet clutch and established a calculation model for the engagement torque of the multidisc wet clutch. The clutch engagement torque was simulated in different lubrication stages. This study has a theoretical significance for the friction characteristics of wet clutches. Nevertheless, the engagement torque model cannot be used in clutch control because of its nonlinearity. Zhang $\mathrm{H}$. et al. [13] analyzed the friction torque attenuation of a wet multidisc clutch in the slip phase. $\mathrm{Yu} \mathrm{L}$. et al. [14] established the calculation model of the average specific pressure and torque within the interfaces of multifriction pairs, obtained the attenuation coefficient of the specific pressure of the multifriction pairs, and analyzed the sensitivities of the parameters that affected the specific pressure attenuation for the clutch by considering the frictional force of the spline. Cho J. et al. [15] analyzed the frictional torque during the wet clutch engagement, considering sliding contact between the friction disc and the steel separator. Hu H. W. et al. [16] constructed a state space equation of the tracking control system based on a clutch actuator model and designed the sliding mode controller to improve the tracking control quality of the clutch actuator during engagement. However, the established state space model was complicated; hence, its application in practical engineering was difficult. Balau E. A. et al. [17] performed the dynamic modeling of an electrohydraulic wet clutch system and proposed a model-based predictive control strategy to control the displacement of clutch piston and reduce the effect of delay induced by networking on control performance. However, only the tracking response of the control strategy was analyzed. In addition, the control strategy with high complexity was difficult to achieve in practical application. Jian H. C. et al. [18] proposed an optimized design method of a wet clutch's pressure control valve aiming at the instability that aroused from self-excited vibration caused by the inherent nonlinearity and fluid dynamic coupling of the electrohydraulic valve. Dutta A. et al. [19] reviewed clutch control strategies, including modelbased (e.g., nonlinear model predictive control, iterative learning control, and iterative optimization) and model-free strategies (e.g., genetic-based machine learning and reinforcement learning). However, the implementation of these strategies in practical engineering applications is difficult because of complexity and nonlinearity. Jung S. H. et al. [20] proposed a new pressure hysteresis model of an electrohydraulic actuator for wet clutch engagement based on physical phenomena. A feed-forward controller for hysteresis compensation was designed on the basis of the proposed model, and an additional feedback controller was applied to control the stability of the time-delay system. Yu H. C. et al. [21] designed a nonlinear controller based on backsteppping control method through a nonlinear model for an electrohydraulic valve for wet clutch, which realized the hydraulic tracking control of clutch pressure. Large errors between actual and target pressure were found during dynamic verification because the time delay of the system response caused by hysteresis was not considered. Yu Z. L. et al. [22] modeled the hysteresis nonlinearity of a piezoelectric ceramic actuator via the improved PrandtlIshlinskii (PI) model. A feed-forward inverse compensation control method in combination with PID was established on the basis of the proposed model to realize the nonlinear hysteretic control of the actuator. This method essentially depended on the accuracy of the model, and input signal changes or environmental factors in actual engineering applications would reduce compensation accuracy. Similarly, Zhao T. et al. [23] modeled the hysteresis creep nonlinearity of a piezoelectric ceramic laminated actuator using the improved PI model, and obtained the hysteresis inverse compensation model combined with the adaptive filtering feedback control to regulate the input signals in real time. They realized the hysteretic creep self-adaptive control for the actuator. Although this method obtained high nonlinear tracking accuracy of the hysteresis creep, it still depended on the accuracy of the inverse model. Tao Y. D. et al. [24] constructed a rate-related hysteresis nonlinearity model for a piezoelectric actuator and carried out the hysteresis compensation control based on the proposed model and the Gaussian process (GP).

The aforementioned research results mainly focused on transmission torque estimation, friction characteristic analysis, and hydraulic system control for wet clutches. Few studies on hysteresis characteristics and control are also explored. Studies on wet clutch control are mostly conducted 
using model-based methods, thereby resulting in certain improvements in delay suppression and accuracy control for pressure response. However, model-based methods depend on the accuracy of the established models. Moreover, the electrohydraulic valve in a wet clutch system shows remarkable nonlinear characteristics. Thus, realizing the model-based methods in practical engineering applications is difficult. In addition, hysteresis models are usually employed in hysteresis compensation control. With high nonlinearity, the control algorithms are complicated and not suitable for pressure hysteresis control for wet clutches. Taking a wet clutch in DCT as research object, this study theoretically and experimentally analyzes the hysteresis characteristics of the electrohydraulic pressure control valve and establishes a compound controller of feed-forward compensation controller with additional feedback controller. Bench tests are conducted using different control methods under various working conditions to analyze the control effects of the proposed method. This study can provide an effective control method for pressure hysteresis compensation with high precision for the wet clutches of vehicles.

The remainder of this work is organized as follows. Section 3 describes the working principle of the wet clutch's electrohydraulic pressure control valve, theoretically and experimentally analyzes the pressure hysteresis, and proposes the compound control method of feed-forward compensation control with additional PID based on hysteresis features. Section 4 discusses the pressure tracking control effects through bench tests. Section 5 summarizes the conclusions.

\section{Methodology}

\subsection{Working principle of the pressure control valve}

Wet clutches are core components of DCTs. DCTs use two sets of wet clutch systems to achieve engagement. Given that one clutch is separated with the other one engaged, the power interruption during shifting can be avoided, and excellent dynamic performance can be guaranteed. The pressure of the hydraulic system controlled by pressure control valve determines the separation and engagement of the wet clutch. Fig. 1 shows that the pressure control valve of the wet clutch is composed of an electrohydraulic proportional solenoid valve, a pressure sensor, and a pillar spool. Electromagnetic force is generated by adjusting the control current of the solenoid, thereby resulting in the movement of the spool. During the movement of the spool, the positions and conduction areas of the fluid inlet and outlet will alter. The former controls the direction of the fluid flow, whereas the latter adjusts the pressure of the hydraulic oil that acts on the clutch. The pressure sensor detects the output pressure of the clutch in real time and feeds back to the transmission control unit (TCU), which is used to control the hydraulic system. After the clutch pressure is established by the control system, the engine torque can be transmitted to the power train via the frictional forces that act on the frictional discs of the wet clutch, thereby driving the vehicle to speed up and slow down.

The spool is inevitably subjected to the damping forces during its movement because of the hydraulic oil medium. Given that the movement is reciprocating, the damping force direction will alter with the motion direction, thereby generating hysteresis in the pressure control valve. Hysteresis causes response delay in the hydraulic system, which in turn affects the clutch pressure control accuracy.
Therefore, optimized clutch pressure control will play a key role in improving the starting performance of the vehicle, enhancing the shift quality, and boosting the torsional vibration effect.

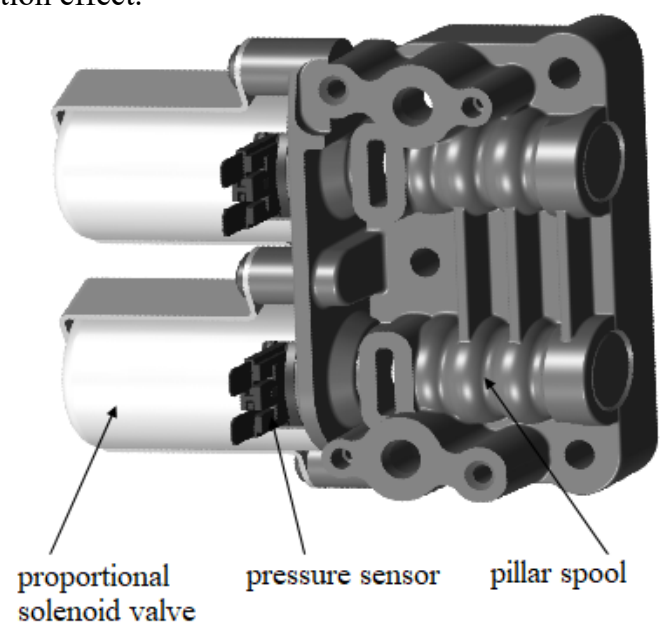

Fig. 1. Electrohydraulic pressure control valve

\subsection{Analysis of the pressure hysteresis}

The pressure hysteresis of the clutch is generated during the movement of the spool. The mathematical analysis of the spool is carried out in this part. The spool is subjected to a combination of forces, including the electromagnetic force generated by the solenoid, the preload of the return spring, the hydraulic force at steady state, the transient impact force, and the damping force caused by the hydraulic oil. According to Stribeck theory [25-26], the damping force is related to various factors, such as oil temperature, spool material and movement speed, and normal forces. As the movement speed of the spool increases, the lubricating state changes, and the damping force can be simplified from Coulomb friction force to mixed friction force. When the speed rises to a certain extent, the lubricating state varied to hydrodynamic lubrication, and the damping force can be treated as a viscous damping force. Considering the response speed requirement of the clutch pressure, the spool maintains a relatively high speed. Thus, mixed friction forces are adopted to describe the damping state and ignore the unstable impact force that acts on the spool. Therefore, the motion of equation (EOM) of the spool can be expressed as follows:

$$
F_{a}=m \ddot{x}+\left(F_{f}+\left(c_{v}+c_{n}\right) \dot{x}\right) \operatorname{sign}(\dot{x})+\left(k_{v}+k_{s}\right)\left(x+x_{0}\right)
$$

where $F_{a}$ represents of the electromagnetic force of the solenoid, N. $m$ denotes the spool mass, $\mathrm{kg} . c_{v}$ and $c_{n}$ are the transient hydrodynamic coefficient and the equivalent viscous damping coefficient, $\mathrm{N} /(\mathrm{m} / \mathrm{s})$, respectively. $k_{v}$ and $k_{s}$ describe the steady-state hydrodynamic coefficient and the stiffness of the support spring, $\mathrm{N} / \mathrm{m}$, respectively. $x$ indicates the spool displacement, m. $x_{0}$ refers to the compression deflection of the support spring under preloading state, $\mathrm{m} . F_{f}$ denotes the Coulomb friction force that acts on the spool, N.

For the magnetic loop, the electromagnetic force of the solenoid can be written by Maxwell's formula as: 
$F_{a}=\alpha \frac{\Phi^{2}}{2 \mu S}=\alpha \frac{B^{2} S}{2 \mu}$

where $\Phi$ is the magnetic flux of the air gap, $\mathrm{Wb}$; and $\Phi=B S . B$ denotes the flux density of the air gap, T, whereas $S$ represents the total area of the electromagnetic air gap. $\mu$ is the air gap permeability, in general, $\mu=4 \pi \times 10^{-7}, \mathrm{H} / \mathrm{m} . \alpha$ indicates a coefficient that depends on the number of magnetic poles.

Given that the magnetic permeability of the ferromagnetic material is greater than that of the air gap, only the effect of the air gap is considered. According to Kirchhoff's second law, the magnetomotive force is expressed as follows:

$$
F=N I=\frac{2 \Phi \delta}{\mu S}=\frac{2 B \delta}{\mu}
$$

where $F$ denotes the magnetomotive force, A. $\delta$ is the maximum air gap, m. $N$ represents the number of the coil turns that generate magnetization field. $I$ indicates the conduction current for generating the magnetization field, A.

Therefore, the EOM of the spool can be rewritten as:

$$
\begin{aligned}
& m \ddot{x}+\left(F_{f}+\left(c_{v}+c_{n}\right) \dot{x}\right) \operatorname{sign}(\dot{x})+\left(k_{v}+k_{s}\right)\left(x+x_{0}\right) \\
& =\frac{\alpha N^{2} I^{2} \mu S}{8 \delta^{2}}
\end{aligned}
$$

When the spool moves, it quickly passes the acceleration and deceleration zones. As the spool moves stably, the speed can be considered constant, and acceleration will be zero. Let $F_{\mu}=F_{f}+\left(c_{v}+c_{n}\right) \dot{x}, C_{1}=\left(k_{v}+k_{n}\right) \dot{x}_{0}=k x_{0} \quad$, and $\beta=\frac{\alpha N^{2} \mu S}{8 \delta^{2}} . F_{\mu}$ represents the total damping force of the spool, N. $C_{1}$ denotes the total elastic force caused by preloading, N. Since $\operatorname{sign}(\dot{x})= \pm 1$, Eq. (4) can be given by

$$
\pm F_{\mu}+k x+C_{1}=\beta I^{2}
$$

Consequently, the relationship between the spool displacement and the control current of the solenoid in steady state can be determined as follows:

$$
x=\frac{\left(\beta I^{2}-C_{1}\right) \mp F_{\mu}}{k}
$$

The spool displacement is related to the control current of the solenoid. The directions of the damping force during the processes of increasing and decreasing the control current are opposite, causing one control current to correspond to two different spool displacements. In addition, the positive motion displacement is less than the reverse one under the same control current.
With tiny spool displacement, the conduction areas of the oil outlet and the drain port are proportional to the displacement. Hence, the above relationship also exists between the clutch pressure and the control current. Similarly, the ascending and descending clutch pressure during the increase and decrease of control current cannot coincide with each other, which forms hysteresis loops. In practical control, the target pressure of the clutch is usually used as the system input, and the control current is given according to the relationship between the clutch pressure and the control current. Consequently, the actual clutch pressure is exported after generating the control current. However, one target pressure corresponds to two different control currents with hysteresis, leading to two actual pressures. Eq. (6) shows that the control current that corresponds to the ascending pressure is lower than that related to the descending pressure. The larger the difference is between the ascending and descending pressures caused by the hysteresis, the greater the deviation is between the actual pressure and the target pressure of the clutch, and the lower the control accuracy is.

\subsection{Bench tests}

Figure 2 shows the test bench for the control of the wet clutch system, which consists of three dynos, the DCT assembly, TCU, the data acquisition device, and the computer. The dynos are controlled by frequency conversion and are automatically adjusted to output different rotational speeds and torques. No. 1 dyno is used as the power input component to the DCT assembly. Nos. 2 and 3 dynos are adopted to simulate different loads for the system. Temperature and pressure sensors are mounted in the hydraulic system of the wet clutches in the DCT assembly, which provide the oil temperature and clutch pressure to TCU. TCU implements DCT control and is used to read detection data. The computer can read the data from TCU through the data acquisition device. The electrohydraulic pressure control valve of the wet clutch studied in this research has a control range of 0-1200 $\mathrm{mA}$ current and can achieve pressure control in range of $0-20$ bar. The technical requirement for the pressure control accuracy of the clutch in vehicle applications should be within 0.1 bar, otherwise it will lead to vehicle crawling and decline of the starting performance and the unevenness of DCT shifting.

In this part, two experiments are designed to systematically study the corresponding relationship between the control current of the electrohydraulic valve and the clutch output pressure, and the test data for the following feed-forward control algorithm are provided.

First, the current with triangular trajectory currents with a step of $100 \mathrm{~mA}$ is applied to the solenoid, of which the peak values are gradually decreased from 1200 to $400 \mathrm{~mA}$ (Fig. 3(a)). Under this current excitation condition, the clutch output pressure can be obtained, and the currentpressure (I-P) curve is plotted in Fig. 4. From this experiment, the maxima of the output pressure of the clutch will be determined. If the output pressure of the clutch is initially low but subsequently increases monotonically, when it reaches a local maximum during the ascending process, the local maximum can be defined as the maximum value of the clutch pressure. 


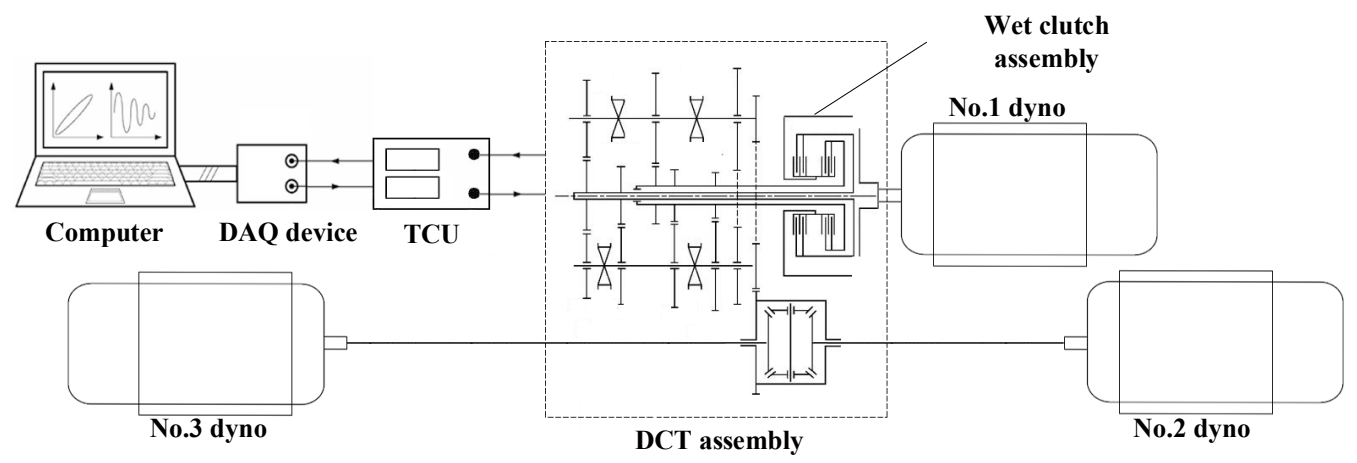

Fig. 2. Test bench

Second, the current with triangular trajectory with a step of $100 \mathrm{~mA}$ is applied to the solenoid, of which the peak value is stable to $1200 \mathrm{~mA}$, but the minimum value is gradually increased (Fig. 3 (b)). Similarly, the I-P curve can be plotted in Fig. 5. The minima of the output pressure of the clutch can be determined from this experiment. If the output pressure of the clutch is initially high but subsequently decreases monotonically, when it reaches a local minimum during this process, the local minimum can be defined as the minimum value of the clutch pressure.

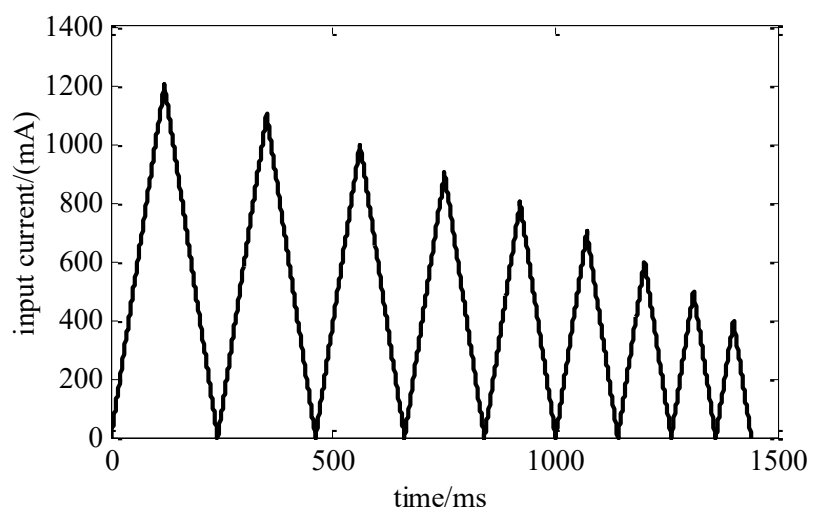

(a)

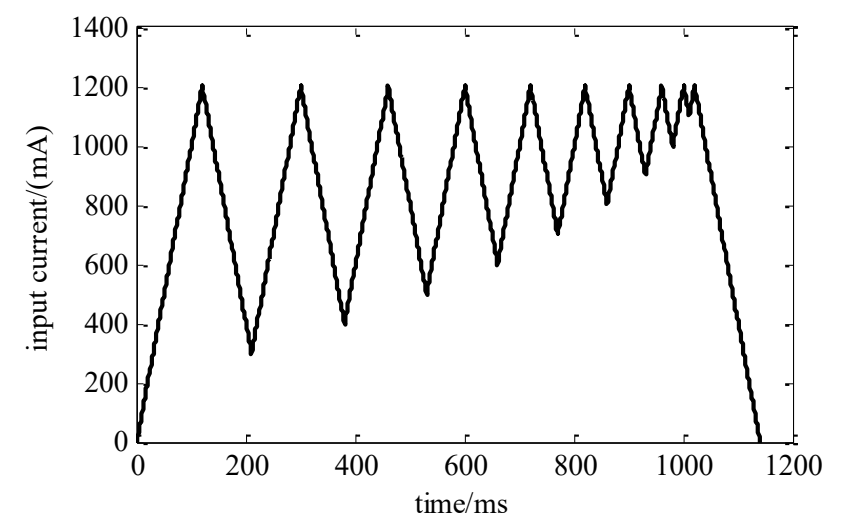

(b)

Fig. 3. Input currents in experimental design. (a) Control current in experiment 1. (b) Control current in experiment 2

Figure 4 depicts the relationship between the input current and the clutch pressure for experiment 1 . Hysteresis exists between the input current and the clutch pressure. The pressure curve that corresponds to the rise of the current is inferior to that related to the fall of the current, which agrees with the results of theoretical analysis. A sequence of maximum values of the clutch pressure can be obtained from the experimental results.
Figure 5 shows the relationship between input current and clutch pressure for experiment 2. Obvious hysteresis occurs between the control current and the clutch pressure. The I-P curve shows that the pressure curve that corresponds to the increase of the current is lower than that related to the decrease of the current, which confirms the results of theoretical analysis again. The sequence of minimum values of the clutch pressure can be obtained from the experimental results.

The I-P curves of both experiments show that the maximum difference of the clutch pressure between the ascending and descending process can reach 0.48 bar, that is, the maximum value of the hysteresis is 0.48 bar. Hysteresis results in the nonunique mapping between the input current and the output pressure during the control process. Since the hysteresis is beyond the accuracy requirement of vehicle control $(<0.1$ bar), establishing compensation control for pressure hysteresis is important.

\subsection{Compensation control algorithm for pressure Hysteresis}

Feed-forward control is a predictive method among the commonly used control methods. Feed-forward control treats target values as input, adjusting the output of the controlled object before the appearance of the deviation, which is characterized by low control precision, great dynamic response performance and easy implementation. Feedback control is a tracking method, which treats the error values as input. Thus, adjusting the output of the controlled object after the appearance of deviation becomes possible. Feedback control methods are demonstrated to have high control precision, whereas certain response delays and complexity exist in control systems. As a classical feedback control method, PID has been widely used in industrial control. The target pressure corresponds to two different input currents because of the hysteresis in the electrohydraulic pressure control valve. Obtaining a satisfactory control effect by merely using error values as system input is difficult for PID control method. The input current should be compensated before the actual pressure exports. Therefore, this study proposes a compound control method that contains a feed-forward controller with additional PID controller. The proposed method uses target and error pressure as inputs of the feed-forward controller and the PID controller, respectively. The feed-forward controller can compensate the error pressure caused by hysteresis. With the advantages of the additional PID controller, the compound control method can guarantee system stability, response speed, and pressure control accuracy. 


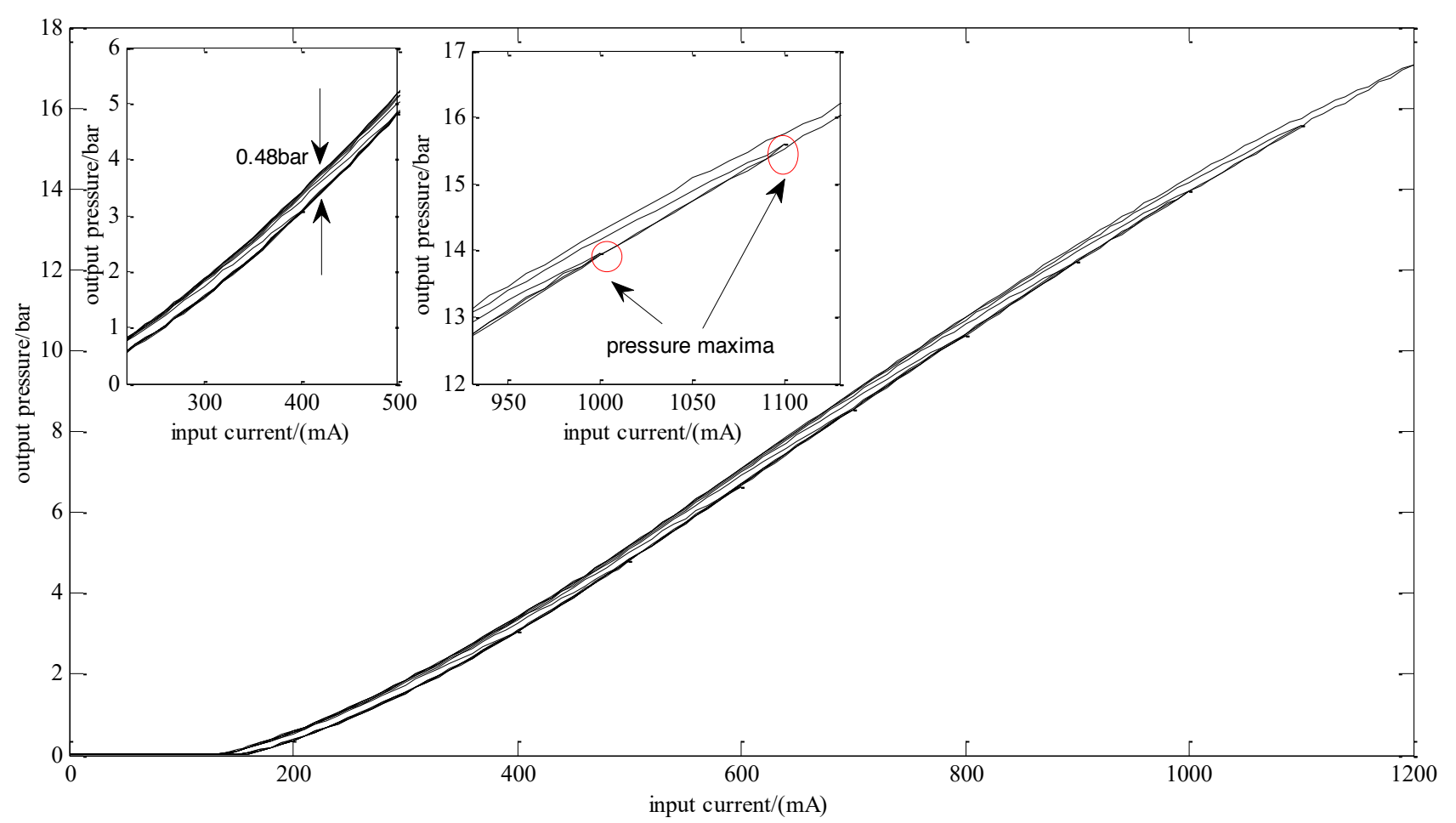

Fig. 4. I-P curve from experiment 1

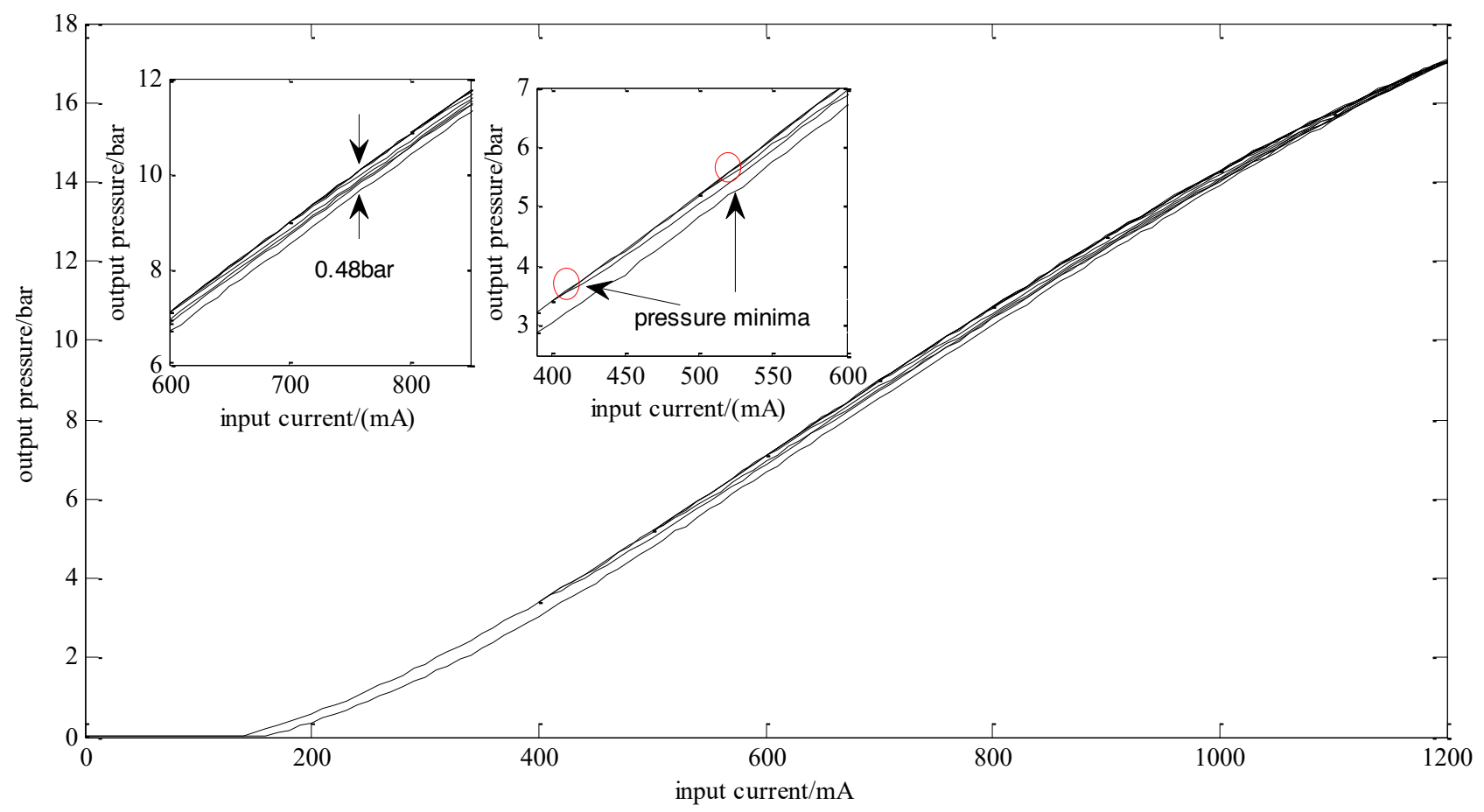

Fig. 5. I-P curve from experiment 2

The feed-forward controller is utilized to compensate the output error pressure caused by hysteresis. In this part, the feed-forward control algorithm is illustrated. The results of the experiments shown in Section 3.2 are used to determine the control parameters of the algorithm. When the control current ranges between $0 \mathrm{~mA}$ to the saturation current, the measured closed I-P curves, including ascending and descending processes, are defined as hysteresis main loops (Figs. 4 and 5). In mass production, certain differences exist among the hysteresis main loops because of the different characteristics presented by the pressure control valve, whereas the deviations between the inner hysteresis loops and the main loops are fixed. Thus, the hysteresis main loops can be treated as the reference of compensation control.

The curve fitting of the curves can be expressed by performing a quadratic polynomial fit for the ascending and descending curves of the hysteresis main loops as follow:

$$
\begin{aligned}
& p=11.618 c^{4}-41.428 c^{3}+50.786 c^{2}-7.245 c+0.162 \\
& p=9.705 c^{4}-37.321 c^{3}+49.914 c^{2}-5.110 c+0.062
\end{aligned}
$$


where $p$ represents the clutch pressure, bar; and $c$ denotes the input current of the electrohydraulic pressure control valve, $\mathrm{mA}$.

Similarly, curve fitting is conducted for inner hysteresis loops. Fig. 6 shows the fitting curves of the hysteresis curves (including main and inner loops) yielded from the bench tests illustrated in Section 3.2.

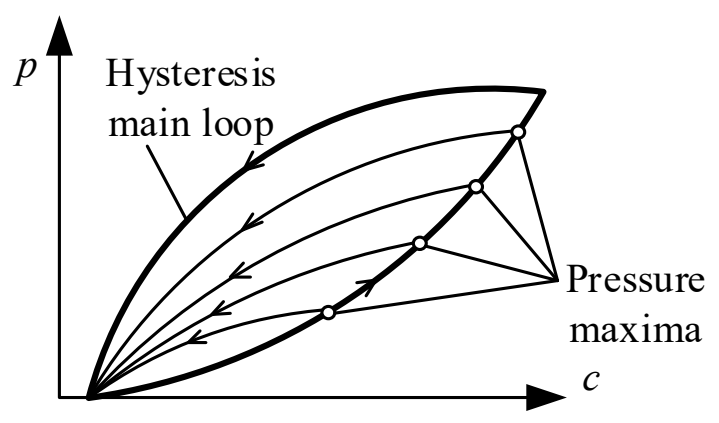

(a)

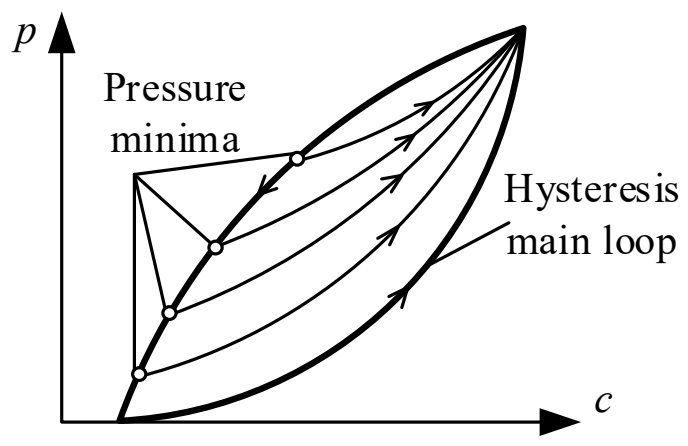

(b)

Fig. 6. Curve fitting of the hysteresis curves. (a) Results from experiment 1. (b) Results from experiment 2

Any inner hysteresis loop can be represented by the hysteresis main loop. Thus, the following formula for describing the relationship between any inner hysteresis loop and the main hysteresis loop can be given by:

$h=s f+t(1-f)$

where $h$ denotes arbitrary inner hysteresis loop. $s$ and $t$ represent the ascending curve and the descending curve of the hysteresis main loop, respectively. $f$ refers to the hysteresis curve coefficient, which is relative to the target pressure and extreme values of the target pressure.

According to the Eqs. (7), (8), and (9) and the extreme values of the output pressure obtained by the results of the experiments illustrated in Section 3.2, the hysteresis curve coefficient $f$ can be calculated and plotted in Figs. 7 and 8 , respectively.

The feed-forward control algorithm includes four items, namely, initialization, extreme values and trend detection, memory and erasing, and calculation and output. Fig. 9 shows the flowchart of the feed-forward control algorithm.

First, the parameter variables are initialized. In this step, $i$ indicates the time sequence with initial value $0 . p_{x}$ represents the maximum value of the target pressure under current working condition, $p_{x}=0$, initially. $p_{y}$ denotes the minimum value of the target pressure under current working condition, with the initial value 0 .

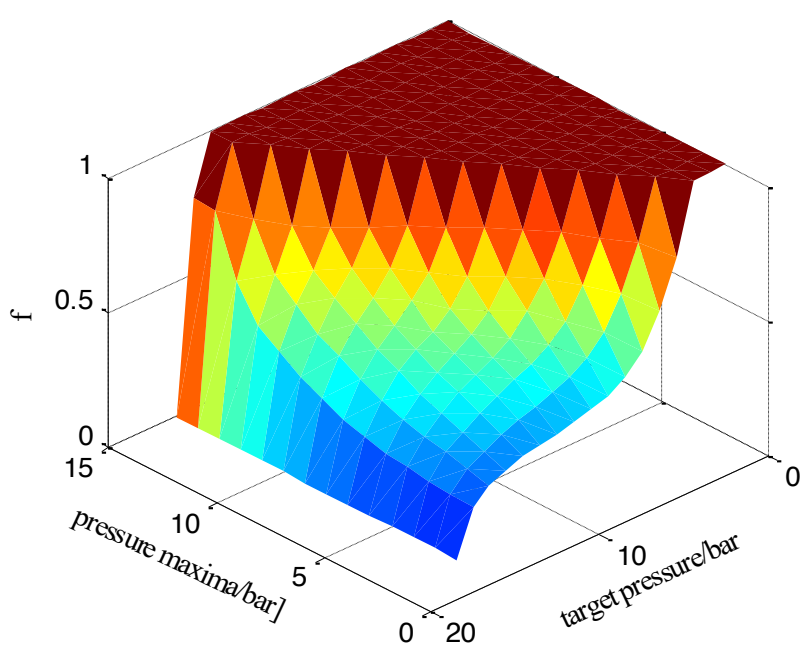

Fig. 7. $f$ corresponding to the pressure maxima

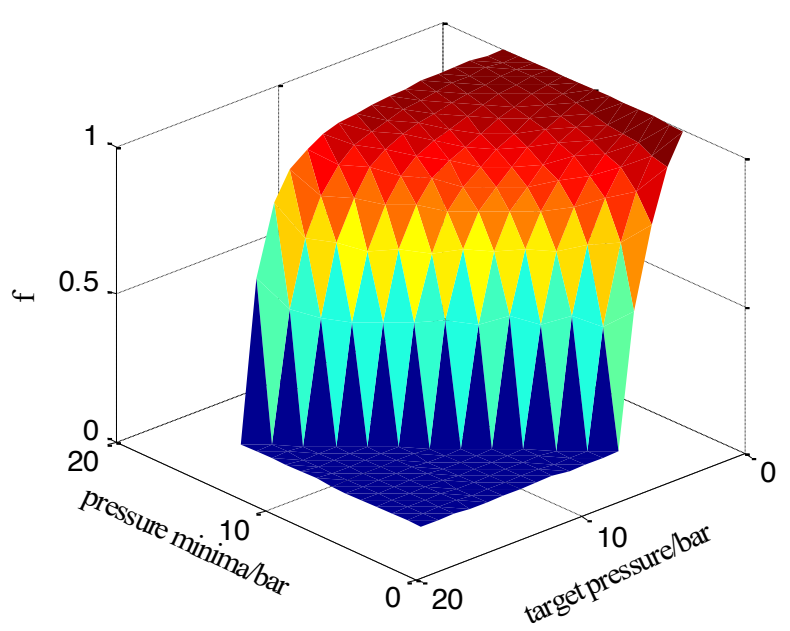

Fig. 8. $f$ corresponding to the pressure minima

Second, the extreme values and the change trend of the target pressure are detected. The change trend is initially determined to identify whether the target pressure is ascending or descending by comparing the pressure of adjacent refresh cycles. After confirming the change trend, the comparison of the target pressure between adjacent refresh cycles is continuously conducted. If the pressure is recognized to decline in the next cycle after its rise in the previous cycle, then the pressure value is the maximum. The pressure minima can be determined in similar approach. In this step, the refresh cycle is indicated by $T$ with the unit of ms.

Third, $p_{x}$ and $p_{y}$ are erased and stored, and the pressure extreme values identified in Step 2 are memorized.

Finally, the control parameters of the feed-forward controller are calculated and exported. Factor_Table $1 / 2$ lists the pressure extreme values and EOL Table indicates the hysteresis main loops. According to Factor_Table1/2 and EOL_Table, control currents $c_{1}$ and $c_{2}$, which correspond to target pressure $p$, can be computed, respectively. Control 
parameters $f_{1}$ and $f_{2}$ can be calculated by conducting interpolation of the target pressure $p$ and extreme pressure $p_{x}$ or $p_{y}$, respectively. Lastly, the control current $c$ can be obtained by using control parameters $f_{1}, f_{2}, c_{1}$, and $c_{2}$

The use of the feed-forward controller ensures that the output can immediately approach the target input, which can reduce the complexity of the feedback controller. To obtain further improvement of control accuracy, the additional PID controller is used after the implementation of the feedforward controller. Fig. 10 shows the block diagram of the compound control model. Through feed-forward control, the basic control current can be obtained through the inputs of the target pressure and pressure extreme values. Then, the actual pressure with compensation exported by the pressure sensor is fed back. The deviation between the actual and the target pressure is used as the input for the additional PID controller to correct the control current of the solenoid. The basic current combined with the corrected current generates the control current for the solenoid of the pressure control valve, thereby generating output pressure with high accuracy. Thus, the compound control method achieves precise pressure control.

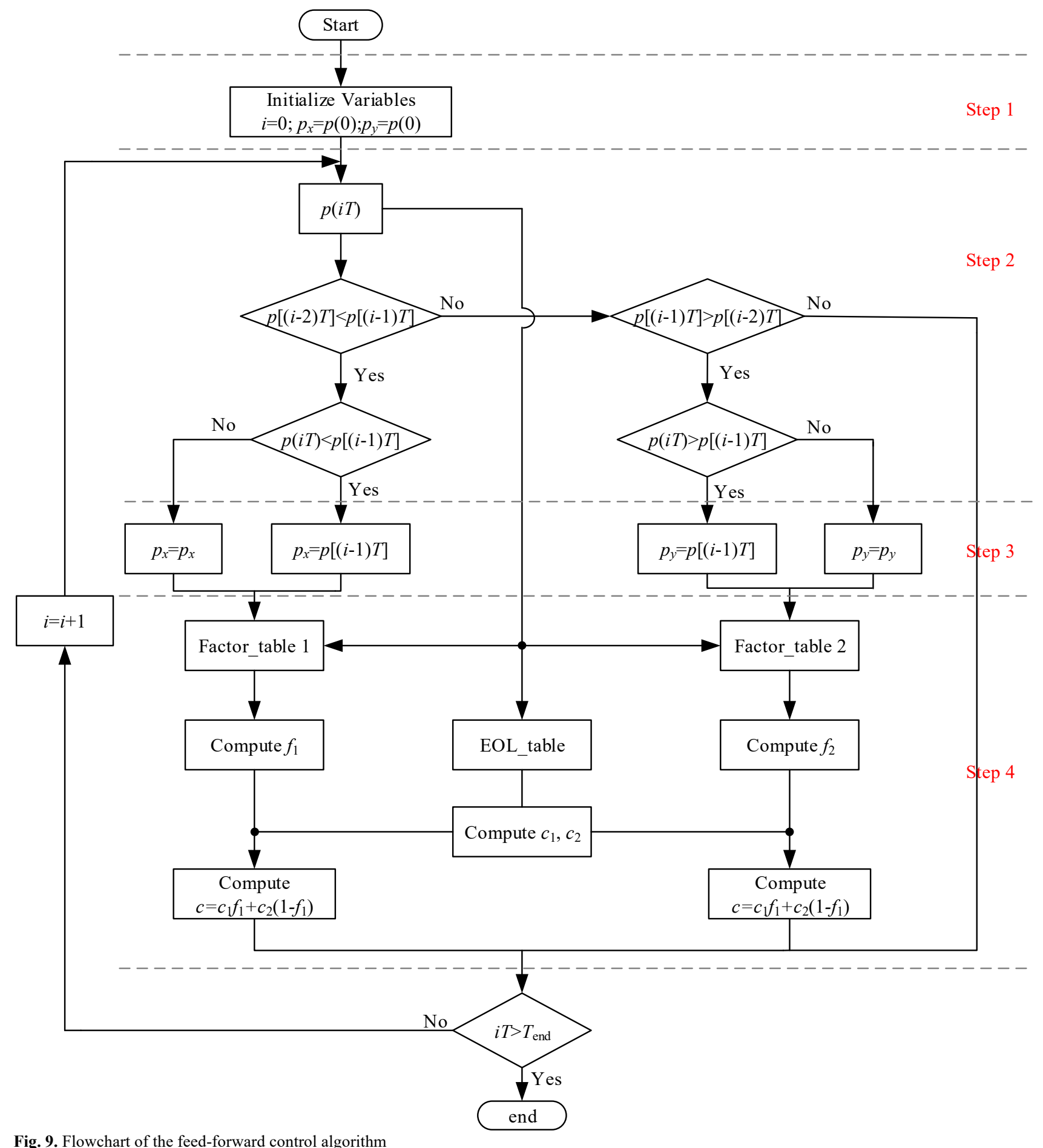

Fig. 9. Flowchart of the feed-forward control algorithm 


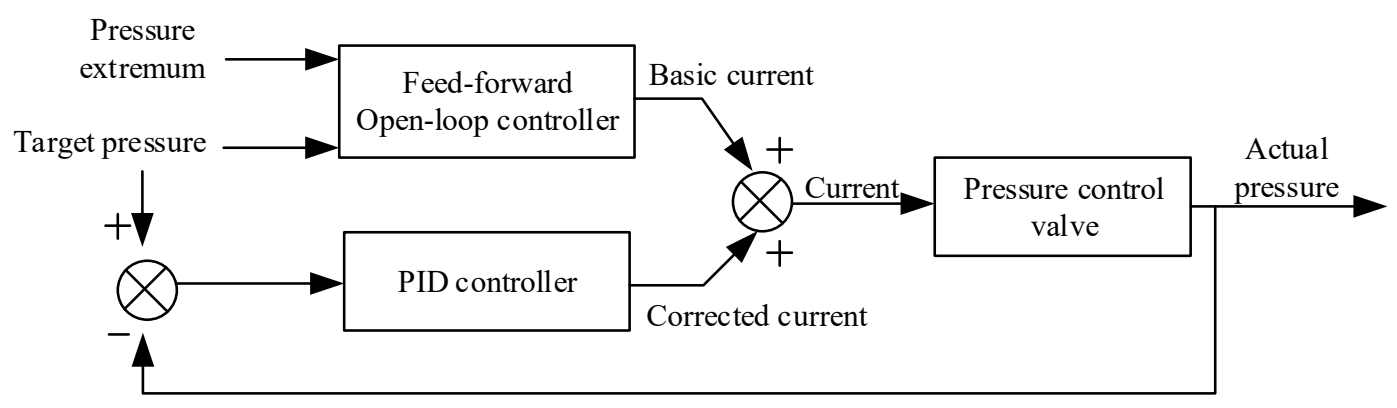

Fig. 10. Block diagram of the compound control model

\section{Results Analysis and Discussion}

In this part, the pressure control method proposed in Section 3.4 is applied to the bench tests to verify the feasibility and accuracy of the proposed method for pressure hysteresis control of the wet clutch. The experimental verification under three different working conditions, namely, steadystate, dynamic, and step pressure tracking, are realized in this section. The pressure tracking effects are also compared using four control methods, namely, the proposed compound control method, the feed-forward control method, the PID control, and the system without compensation control. With pressure hysteresis, the target pressure in the bench tests is controlled from 4 bar to 10 bar. Under the first two working conditions, the target pressures are set up with inflection points at 4 bar and 10 bar, which indicates the minimum and the maximum values of the target pressure, respectively. Under the third working condition, the target pressure jumps from 4 bar to 8 bar.

\subsection{Steady state pressure tracking test}

Steady state refers to the test condition in which the target pressure continues to be stable for a certain period. The test results can reflect the stable pressure tracking accuracy of the control system. Each of the time duration of the steady pressure is set to be $100 \mathrm{~ms}$, and the clutch pressure output by the pressure sensor is recorded. Fig. 11 shows the output pressure, as well as the target pressure, using different control methods. Absolute error is defined as the difference between the target and the actual output pressure. Fig. 12 depicts the absolute errors under the steady state for different control methods. Relative error is defined as the ratio of the absolute error to the target pressure. The maximum pressure deviation, average absolute errors, and average relative errors determined through different control methods are listed in Tab. 1. The test results show the optimal tracking accuracy of the proposed compound control under the steady state, compared with the other control methods. The feedforward control method also represents relatively good performance in pressure tracking, since the hysteresis compensation is involved in this method. PID control method shows poor performance in pressure tracking under steady state, because the hysteresis compensation is not considered in this method. The compound control method can reduce the average absolute error from 0.186 bar to 0.005 bar, thereby meeting the accuracy requirements for practical pressure control.

\subsection{Dynamic pressure tracking test}

Dynamic working condition refers to the test condition in which the target pressure changes linearly with a certain slope. The test results can reflect the dynamic pressure tracking accuracy of the control system. A pressure change slope of 0.65 bar per second is applied in the test. The target and actual pressure curves are plotted separately in Fig. 13 using different control methods. Fig. 14 depicts the absolute tracking errors under dynamic working condition with different control methods. The maximum pressure deviation, average absolute errors, and average relative errors produced by different control methods are described in Tab. 2. The test results show the same pressure tracking effects of the different control methods under dynamic state as that under steady state. The proposed compound control method also represents the optimal performance in pressure tracking. According to Tab. 2, it can reduce the average absolute error from 0.189 bar to 0.043 bar with respect to the uncompensated control, and the average relative error decreases from $2.963 \%$ to $0.722 \%$, which also indicates that the compound control method can meet the accuracy requirements for actual pressure control.

\subsection{Pressure tracking test under step input}

Step pressure tracking is used to measure the pressure tracking effects of different control methods for step input. During the test, the target pressure leaped quickly from 4 bar forward to 8 bar, after that the target pressure get stable at 8 bar. Fig. 15 depicts the curves of the actual output pressure using the four control methods as well as the target pressure. The test results show that the proposed compound control method has no significant improvement in response speed. Compared with the uncompensated control, no obvious difference exists in the first three control methods as to the response time for the pressure leaping to the steady state, since all of them can guarantee relatively high response speed. In addition, as PID control method can reduce system uncertainties, the compound control and the PID control can ensure that the final actual pressure tends to the target pressure, whereas the actual pressure by means of feedforward and uncompensated control can not approach the target pressure. Therefore, under the step input, from the perspective of control accuracy, the compound control method can guarantee the highest control accuracy, followed by PID control, feed-forward control, and finally uncompensated control. 


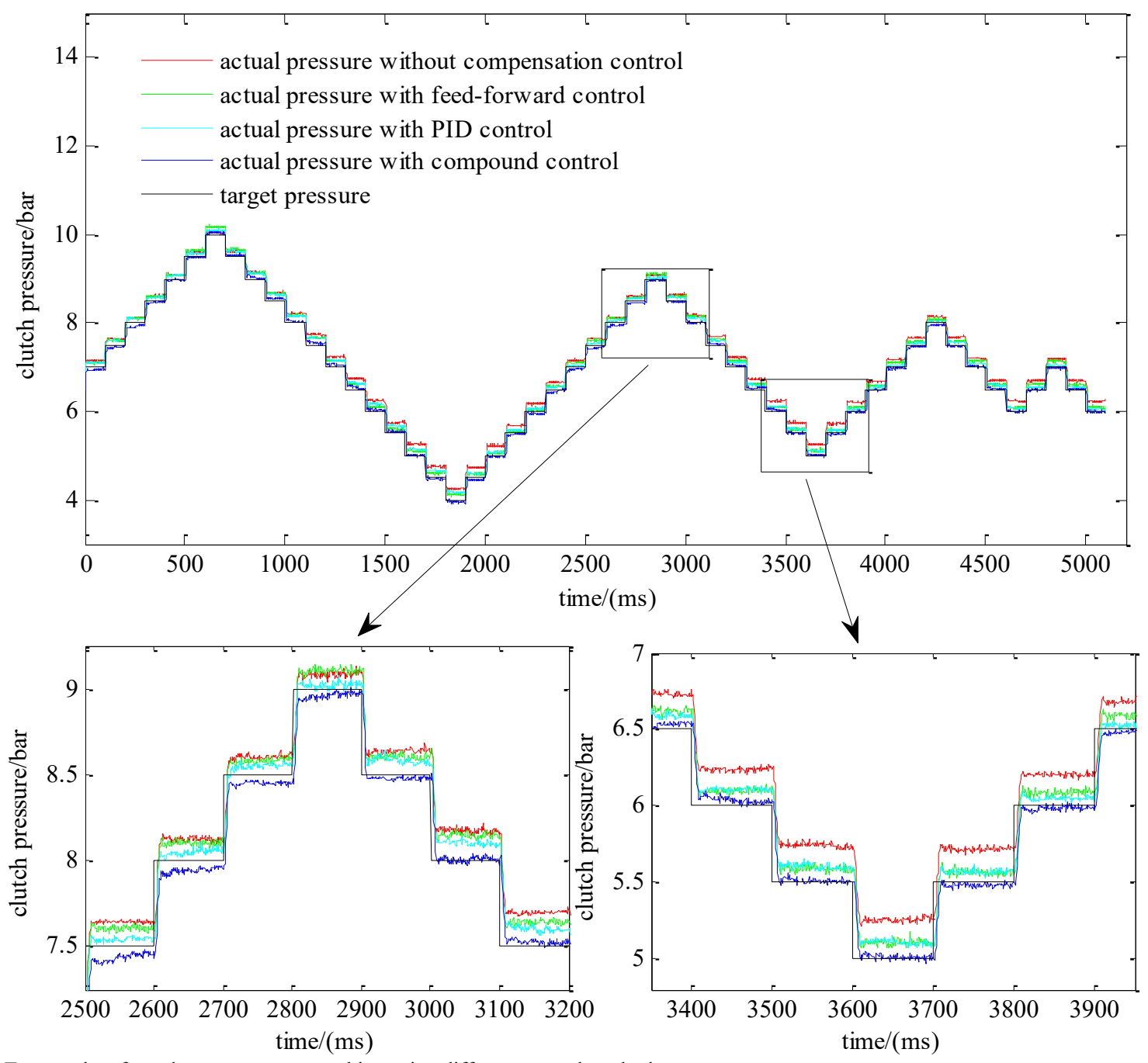

Fig. 11. Test results of steady state pressure tracking using different control methods

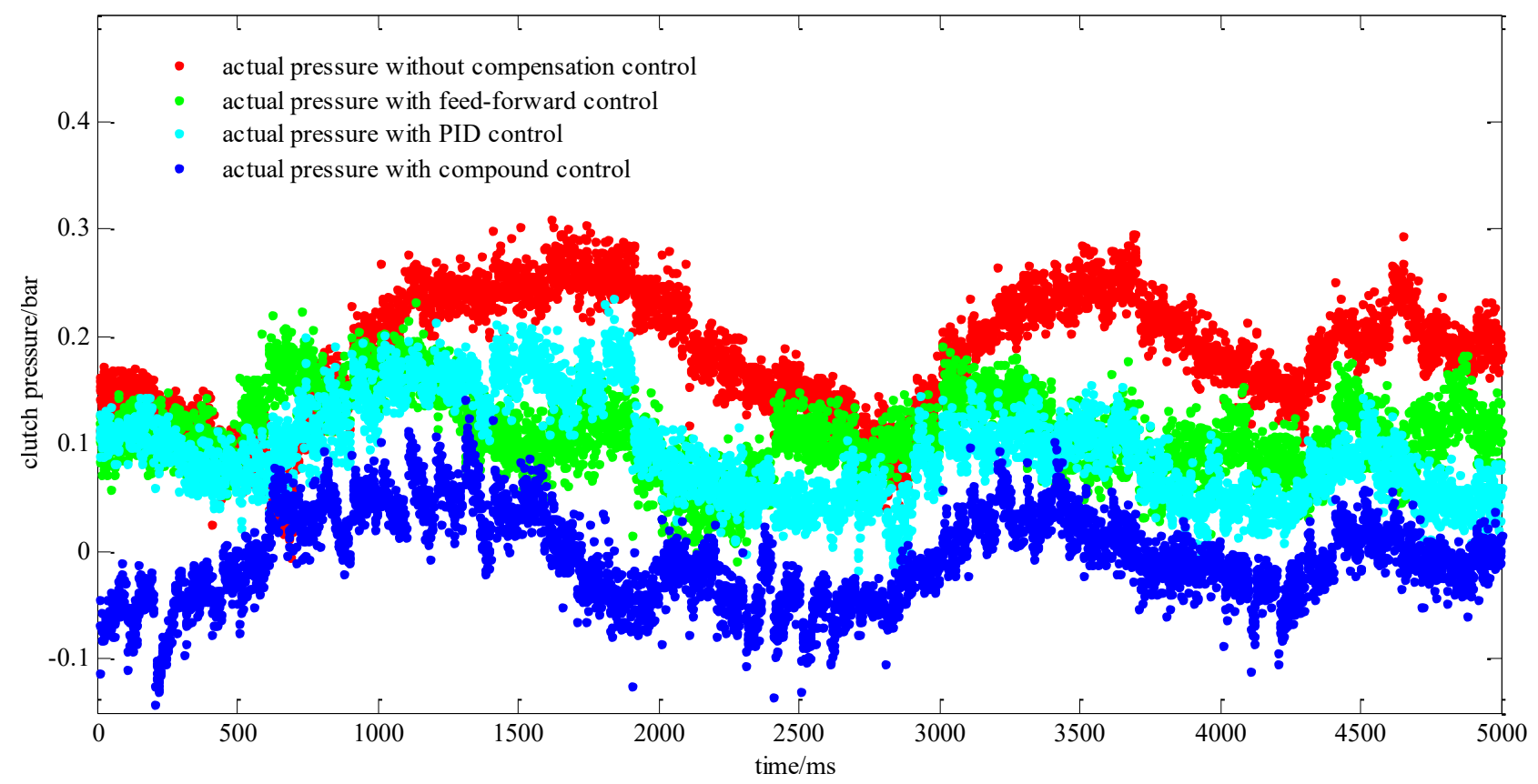

Fig. 12. Absolute tracking errors under steady state 

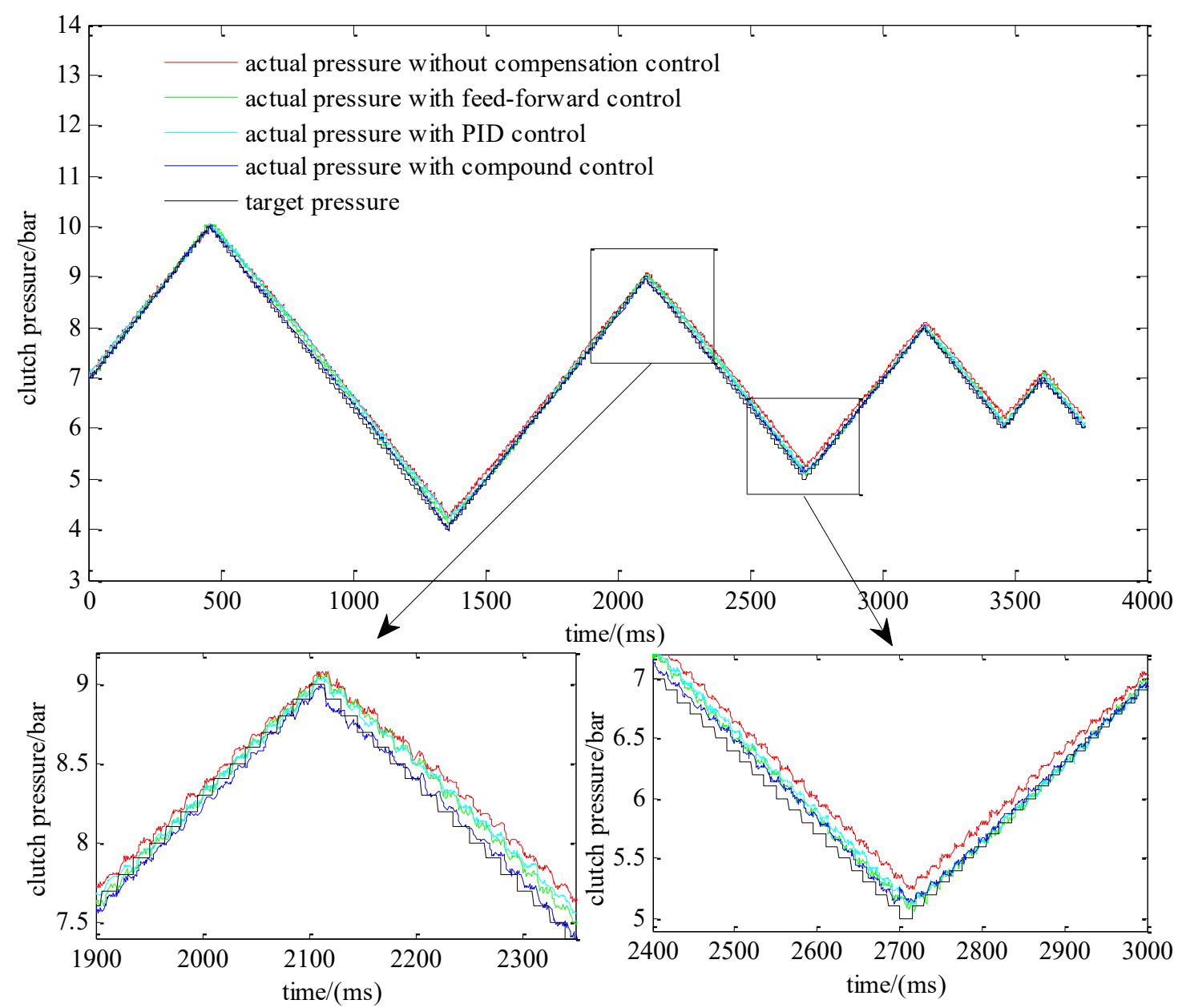

Fig. 13. Test results of dynamic pressure tracking using different control methods

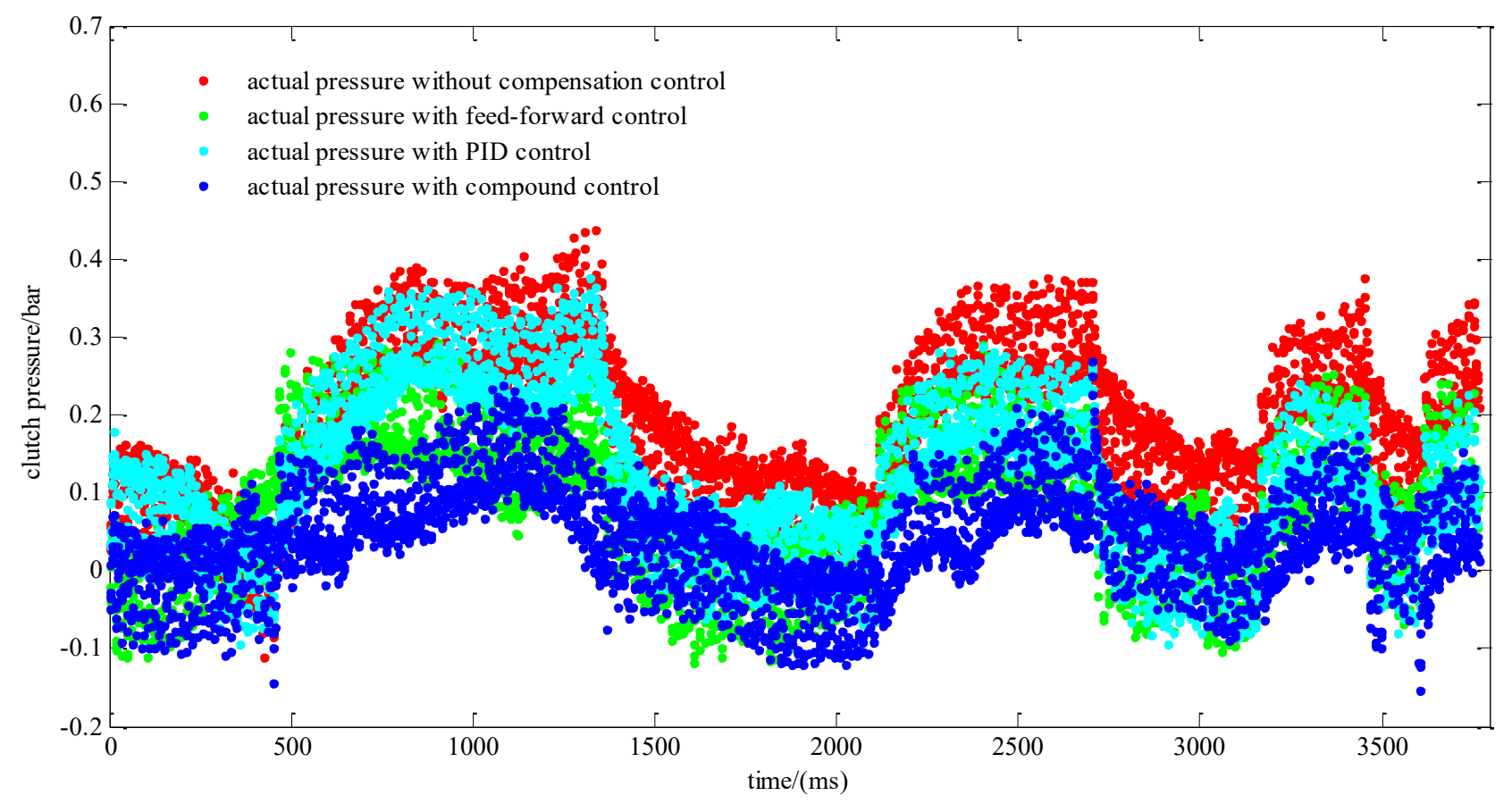

Fig. 14. Absolute tracking errors under dynamic working condition 


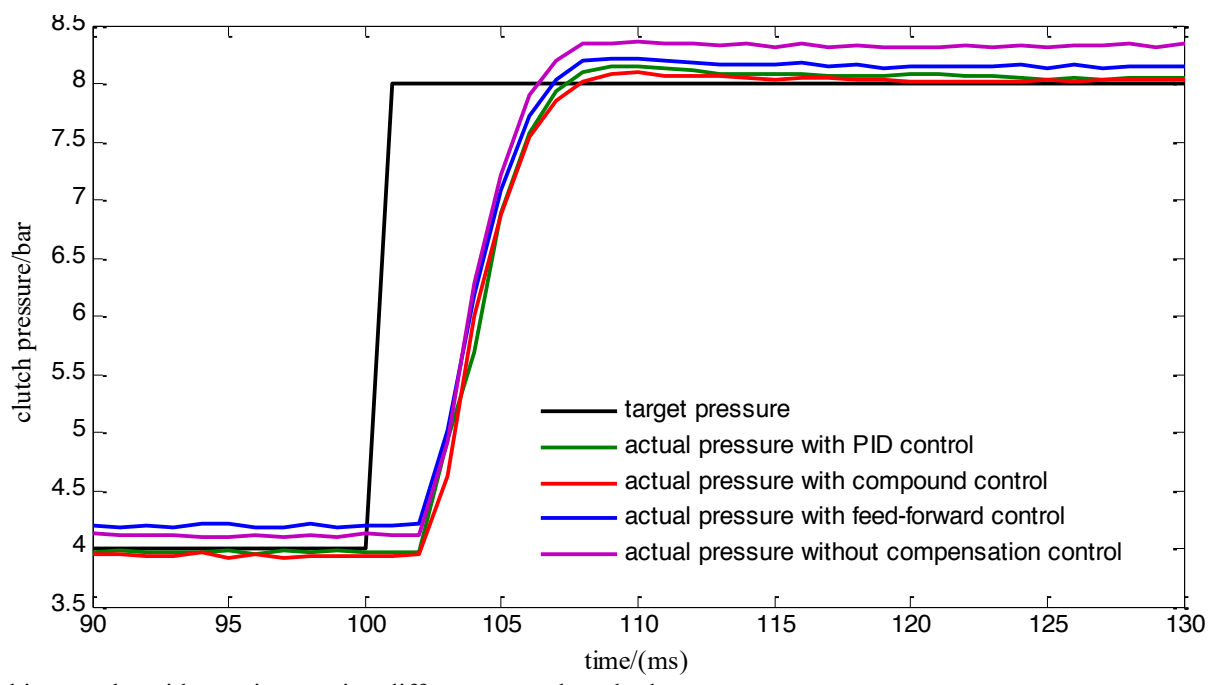

Fig. 15. Pressure tracking results with step input using different control methods

Table 1. Steady state pressure tracking errors using different control methods

\begin{tabular}{c|c|c|c}
\hline Control methods & Maximum deviation/bar & Average absolute error/bar & Average relative error/\% \\
\hline No compensation control & 0.321 & 0.186 & 2.924 \\
Feed-forward control only & 0.233 & 0.108 & 1.616 \\
PID control only & 0.236 & 0.113 & 1.434 \\
Compound control & 0.141 & 0.005 & 0.092 \\
\hline
\end{tabular}

Table 2. Dynamic pressure tracking errors using different control methods

\begin{tabular}{c|c|c|c}
\hline Control methods & Maximum deviation/bar & Average absolute error/bar & Average relative error/\% \\
\hline No compensation control & 0.428 & 0.189 & 0.091 \\
Feed-forward control only & 0.326 & 0.121 & 1.344 \\
PID control only & 0.377 & 0.043 & 1.877 \\
Compound control & 0.182 & 0.722 \\
\hline
\end{tabular}

\section{Conclusions}

To solve the problems of inaccurate pressure control and pressure response delay caused by the hysteresis of the wet clutch's electrohydraulic pressure control valve in DCT, an effective approach for hysteresis compensation control was proposed on the basis of the hysteretic features of the electrohydraulic pressure control valve. Mathematical analysis combined with experimental studies was conducted to characterize hysteresis. Bench tests were facilitated to verify the pressure tracking effects of the proposed control method. The following conclusions could be drawn:

(1) Under steady state, the proposed compound control method can significantly improve the pressure tracking accuracy. In contrast with the system without any compensation control, the compound control method reduces the average absolute error of the system from 0.186 bar to 0.005 bar.

(2) Under dynamic control condition, the pressure tracking accuracy is evidently enhanced by the compound control approach. The average absolute error of the system using the compound control method decreased from 0.189 bar to 0.043 bar, in comparison with the system without any compensation control.

(3) Under the state with step pressure input, the feedforward controller method combined with PID controller guarantees the response speed and control accuracy of the system.

(4) The compound control method is better than the feed-forward control or PID control in terms of pressure tracking accuracy and response speed. The feed-forward control has higher control precision than PID control under dynamic and steady states. However, it is not as good as the PID control under the state with step pressure input.
This study proposed a novel method for pressure hysteresis control of wet clutches based on theoretical analysis and experimental design. The established control algorithm further improved the pressure tracking accuracy of the wet clutch and can meet the actual requirements of pressure control, thereby indicating its practical significance for DCT shifting control. The feed-forward control algorithm in the proposed compound control method is only suitable for general driving conditions because it needs to determine the control parameters according to the experimental results. Under low temperature conditions, the viscosity of the hydraulic oil increases exponentially with the decrease in temperature. Exceedingly high viscosity extremely slows down pressure response speed. When the feed-forward control is applied under low temperature and complicated working conditions, the continuous changes in control parameters can lead to irregular fluctuations in output pressure, thereby resulting in driving unevenness and impact of the vehicle. Therefore, the future research should combine the pressure characteristics under low temperature conditions with the proposed model to further revise the feed-forward control algorithm to obtain precise pressure control for wet clutches under more practical working conditions.

\section{Acknowledgements}

This work was supported by the National Natural Science Foundation of China (Grant No. 51605182) and the Fundamental Research Funds for Central Universities (Grant No. 2662016QD001).

This is an Open Access article distributed under the terms of the Creative Commons Attribution License

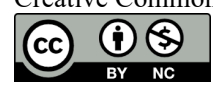




\section{References}

1. Niu, M. K., Ge, A. L., Jin, L., et al., "Introduction of Dual Clutch Transmission". Automotive Technology \& Material, 12, 2002, pp. 36-38.

2. Fu, Y. X., Liu, Y. H., Cui, L. Y., et al., "Dynamic analysis and control strategy of wet clutches during torque phase of gear shift". Journal of Mechanical Science and Technology, 30(4), 2016, pp.1479-1496.

3. Mesmer, F., M., Kiltz, L., Graichen, K., "Model Design for a Hydraulic Clutch Actuation System". International Federation of Automotive Control (IFAC)-paper online, 51(2), 2018, pp. 67-72.

4. Arndt, T., Tarasow, A., Bohn, C., et al., "Estimation of the Clutch Characteristic Map for Wet Clutch Transmissions Considering Actuator Signal and Clutch Slip". International Federation of Automotive Control (IFAC)-paper online, 49(11), 2016, pp. 742748.

5. Ouyang, T. C., Li, S. Y., Huang, G. C., et al., "Mathematical modeling and performance prediction of a clutch actuator for heavy-duty automatic transmission vehicles". Mechanism and Machine Theory, 136, 2019, pp. 190-205.

6. Ompusunggu, A. P., Sas, P., Brussel H. V., "Modeling and simulation of the engagement dynamics of a wet friction clutch system subjected to degradation: An application to condition monitoring and prognostics". Mechatronics, 23(6), 2013, pp. 700712.

7. Karam, M. E., Jiao, Z. X., Zhang, H. Q., "PID controller optimization by GA and its performance on the electro-Hydraulic Servo Control system". Chinese Journal of Aeronautics, 21(4), 2008, pp. 378-384.

8. Losero, R., Lauber, J., Guerra, T. M., "Virtual strain gauge based on a fuzzy discrete angular domain observer: Application to engine and clutch torque estimation issues". Fuzzy sets and systems, 343, 2018, pp. 76-96.

9. Jiwon, J. O., Seibum, B. C., Jinsung, K., "Driveline modeling and estimation of individual clutch torque during gear shifts for dual clutch transmission". Mechatronics, 24(5), 2014, pp. 449-463.

10. Tarasow, A., Bohn, C., Vinaske, M., et al., "Model-based estimation of the torque characteristics of an automated dual-clutch transmission in wet technology". In: 6th IFAC Symposium on Advances in Automotive Control, Munich, Germany: IFAC, 2010, pp. 455-460.

11. Berkel, K. V., Hofman, T., Serrarens, A., et al., "Fast and smooth clutch engagement control for dual-clutch transmissions". Control Engineering Practice, 22, 2014, pp. 57-68.

12. Chen, M., Ma, B., Li, G. Q., et al., "Study on torque characteristics of multi-plate wet clutches during engagement". Journal of Huazhong University of Science and Technology (Natural Science Edition), 42(5), 2014, pp. 34-39.

13. Zhang, H., Li, H. Y., Cang, H., et al., "Experimental study on attenuation characteristics of friction torque transferred by the wet multi-disc clutch". Journal of Harbin Institute of Technology, 50(7), 2018, pp. 94-102.
14. Yu, L., Li, H. Y., Ma, B., et al. "Analysis and verification for average axial pressure attenuation of multi-disc clutch". Journal of Jilin University (Engineering and Technology Edition), 48(4), 2018, pp. 990-997.

15. Cho, J., Lee, Y., Kim, W. J., et al. "Wet Single Clutch Engagement Behaviors in the Dual-Clutch Transmission System". International Journal of Automotive Technology, 19(3), 2018, pp. 463-472.

16. Hu, H. W., Wu, H. Q., Zhang, Z. Y., et al. "Research on trajectory tracking control for wet clutch engagement based on SMC". Procedia Engineering, 15, 2011, pp. 2742-2746.

17. Balau, E. A., Caruntu, F. C., Lazar, C., "Simulation and control of an electro-hydraulic actuated clutch". Mechanical Systems and Signal Processing, 25, 2011, pp. 1911-1922.

18. Jian, H. C., Wei W., Li H. C., et al., "Optimization of a pressure control valve for high power automatic transmission considering stability". Mechanical Systems and Signal Processing, 101, 2018, pp. 182-196.

19. Dutta, A., Zhong, Y., Depraetere, B., et al., "Model-based and model-free learning strategies for wet clutch control". Mechatronics, 24, 2014, pp.1008-1020.

20. Jung, S. H., Choi, S. B., Ko, Y. H., et al., "Pressure control of an electro-hydraulic actuated clutch via novel hysteresis model". Control Engineering Practice, 91, 2019, pp. 104-112.

21. Yu, H. C., Liu, Q. F., Chen, H., "Nonlinear Pressure Control for Clutch Eletro-Hydraulic Control Valve of DCT". Journal of Jilin University (Information Science Edition), 32(5), 2014, pp. 484-492.

22. Yu, Z. L., Wang, Y., Cao, K. R., et al., "Hysteresis compensation and composite control for piezoelectric actuator". Optics and Precision Engineering, 25(8), 2017, pp. 2113-2120.

23. Zhao, T., Yang, Z. C., Liu, H., et al., "Hysteresis and creep nonlinearities modeling and adaptive hybrid compensation control of piezoelectric stack actuators". Acta Aeronautica et Astronautica Sinica, 39(12), 2018, pp. 1-11.

24. Tao, Y. D., Li, H. X., Zhu, L. M., "Rate-dependent hysteresis modeling and compensation of piezoelectric actuators using Gaussian process". Sensors and Actuators A, 295, 2019 pp. 357365.

25. Brandao, J. A., Meheux, M., Ville, F., et al., "Experimental Traction and Stribeck Curves of Mineral, Pao and Ester Based Fully Formulated Gear Oils". In: The Third International Conference on Integrity, Reliability and Failure, Bradford, UK: Emerald, 2009, pp. $1-14$.

26. Marie, M. M. and Tanaka, D. K., "Consideration of Stribeck Diagram Parameters in the Investigation on Wear and Friction Behavior in Lubricated Sliding". Journal of the Brazilian Soceity of Mechanical Science and Engineering, 29 (1), 2007, pp. 56-62. 